\title{
Professor Barry Allen's deep footprints in space, time and spirit
}

\author{
Chen-Yu Huang ${ }^{1} \cdot$ Eva Bezak $^{2} \cdot$ Tomas Kron $^{3} \cdot$ Richard Dove $^{4}$
}

Published online: 4 February 2020

๑) Australasian College of Physical Scientists and Engineers in Medicine 2020

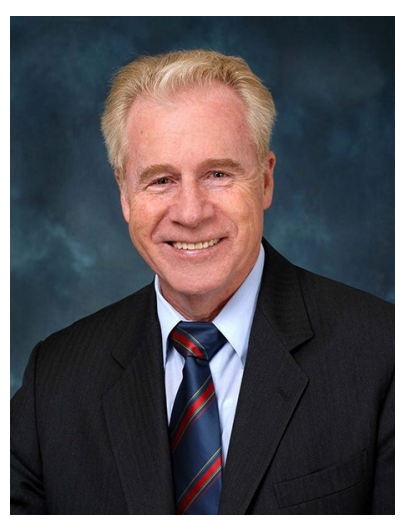

Professor Barry Allen was a distinguished scientist who made weighty contributions to medical physics, biomedical physics and nuclear medicine, and developed innovative methods of cancer treatment. He was also a renowned leader by serving international professional scientific organizations. Professor Allen passed away on 21st November 2019 at the age of 79 due to multiple myeloma.

The authors of this article would like to introduce the significant life of Professor Barry Allen based on his autobiographical speech of "a not so random walk through space, time and spirit" on the investiture of his Order of Australia award in 2015, which is one of the highest awards bestowed in Australia.
Chen-Yu Huang

cyhuangsysu@gmail.com

1 Medical Physics and Research Department, Hong Kong Sanatorium and Hospital, Hong Kong SAR, China

2 University of South Australia, Adelaide, Australia

3 Peter MacCallum Cancer Centre, Melbourne, Australia

4 Canterbury District Health Board, Canterbury, New Zealand

\section{The beginning of Barry Allen's research journey}

Professor Barry Allen's research experience ranges from the formation of radioisotopes in stars to their use in cancer therapy. He completed a BSc in Physics and MSc in Photonuclear Physics with the University of Melbourne (1958-1962).

Following his studies, Professor Barry Allen was subsequently attached to leading nuclear research laboratories throughout the world. He joined the Australian Atomic Energy Commission (AAEC) which later became the Australian Nuclear Science and Technology Organisation (ANSTO) at Lucas Heights in 1963. He married Cynthia and moved to Oak Ridge National Laboratory (ORNL), Tennessee, US in 1969 and later the Central Bureau for Nuclear Measurements (CBNM) in Belgium. Following these stints, he returned to ANSTO in Australia as a Chief Research Scientist (the highest research grade at ANSTO) to do research in high resolution resonance neutron capture. During that time, he published widely in neutron capture reactions, cross section data for fast reactors and investigated neutron capture mechanisms and their relationship to stellar nucleosynthesis (the creation of elements in stars). He published 103 papers in these fields, and he obtained a PhD in resonance neutron capture from the University of Wollongong in 1977, and was later awarded a DSc degree in 1984, the highest degree of the University of Melbourne.

\section{Development of innovative methods in health science}

Professor Barry Allen was an innovator. He opened 'blue sky' research directions by applying technology to medicine with long-term impact. In the early 1980's Professor Allen undertook fundamental research in In Vivo Body Composition (IVBC) measurements for medicine and Boron Neutron Capture Therapy (BNCT) for cancer, making major contributions to both of these fields. 
Professor Allen's work in IVBC was to develop techniques to accurately estimate the proportional percentages of fat relative to protein in the body, which provides useful information for the prognosis of a variety of acute and chronic illnesses.

In the 1980's, Professor Allen designed the first human Body Protein Monitor (BPM) in Australia, which was fabricated by ANSTO at Lucas Heights. Initially for paediatric studies of protein change in cystic fibrosis patients, the BPM was later installed at Royal North Shore Hospital where it continues to operate today. Professor Allen installed a second BPM at the University of Texas Medical Branch in Galveston. A third unit was installed at the Westmead Children's Hospital. Hundreds of paediatric and adult patients with cystic fibrosis, renal disease, cancer and anorexia nervosa have benefited from this work.

Professor Allen's In Vivo Body Composition work made him a major player in the following achievements:

- identification of the importance of enteral nutrition in cystic fibrosis,

- establishment of the role of surgery on body protein,

- determination of lethal levels of total body nitrogen in 'end-stage' renal failure,

- establishment of total body nitrogen threshold levels in cancer chemotherapy,

- identification of the importance of protein replenishment in anorexia nervosa,

- quantification of protein loss in HIV,

- identification of effect of exercise in aging populations.

Professor Allen contributed to over 50 papers which were published in this field.

\section{Development of innovative methods for cancer treatment}

Professor Barry Allen put assiduous effort into the search for a cancer cure. Boron Neutron Capture Therapy (BNCT) has the potential to increase the therapeutic ratio between cancer and normal tissues by using the nuclear reaction between Boron compounds introduced to the tumour and neutron beams. Professor Allen designed the in vivo nude mouse irradiation facility at the Moata reactor, ANSTO, proving for the first time the efficacy of BNCT in the local control of human melanoma in nude mice. He also engineered a thermal beam of neutrons with the reliability and characteristics to be able to be used in clinical therapy. Furthermore, Professor Allen demonstrated for the first time the induction of double strand breaks in DNA arising from neutron capture induced auger emission.
Professor Allen went on to become President of the International Society for Neutron Capture Therapy in 1988 and to convene the Fourth International Symposium in Sydney in 1990.

\section{Leadership in bench to bedside clinical trials}

Professor Allen has shown unique leadership in several research disciplines, some of which have found immediate application in the clinical management of disease. Professor Barry Allen commenced the Targeted Alpha Therapy (TAT) project in 1994 at St George Hospital. TAT links an alpha emitting radioisotope with an antibody which then seeks out cancer cells without damaging healthy tissue. He brought multi-discipline researchers together (in medicine, biology, pathology, chemistry, physics and statistics) and had the ability to 'make things happen'.. His team developed new agents for the treatment of melanoma, leukaemia, breast, prostate, pancreatic, ovarian and colorectal cancers.

Professor Allen successfully brought preclinical TAT studies from bench to bedside. He was the designer and Study Director of two world-first trials. The first trial of intralesional TAT for melanoma commenced in 2001 and recruited 15 patients. A systemic phase I dose escalation trial in 2004 recruited 38 stage 4 melanoma patients. Ten percent of the cohort demonstrated partial response and $30 \%$ had stable disease. These results were superior to other existing approaches and came without significant side effects. One patient had a near complete response, which left him disease free for 12 months. Together with other responses, this achievement was widely reported in the TV news across Australia.

The clinical trial results were Professor Allen's most important papers and gave rise to the Tumour Anti-vascular Alpha Therapy (TAVAT) concept for the regression of solid tumours by Targeted Alpha Therapy. This paper was rated in the top $10 \%$ of papers for 2007 by the journal of Physics in Medicine and Biology, and opens up a new approach to cancer therapy. The paper explains how the short-range alpha radiation emitted by the radioimmunoconjugate can regress solid tumours by targeting perivascular cancer cells. Alpha radiation from these cells creates crossfires along the capillary axis, killing the endothelial cells and closing up the capillary to inhibit tumour growth. The TAVAT concept was also validated by Monte Carlo microdosimetry calculations. For the first time, the possibility of Targeted Alpha Therapy regressing solid cancer tumours was verified by both in silico experiments and clinical trials.

A further important development was the first demonstration of clinical biological dosimetry for systemic Targeted Alpha Therapy, which was based on the formation of micronuclei in lymphocytes randomly hit by alpha emissions in 
the blood. There have been over 70 publications in Targeted Alpha Therapy.

Professor Allen co-authored the text book "Biomedical Physics in Radiotherapy for Cancer" published in 2011 with Drs. Eva Bezak and Loredana Marcu.

Professor Allen's impact on education, post-graduate research, clinical practice and cancer therapy has been substantial at the national and international level. As a result, Professor Allen was awarded Fellowships by

- the Australian Institute of Physics (1972),

- the American Physical Society (1981),

- the Australasian College Physical Scientists \& Engineers in Medicine (1992),

- the Institute of Physics (1999).

Professor Allen's outstanding work was also recognized by the International Organisation of Medical Physics that in 2013 he was named as one of the 50 medical physicists who have made an outstanding contribution to medical physics over the last 50 years. Professor Allen left deep footprints in space and time.

\section{Professor Allen's longstanding contribution to medical physics professional bodies}

Further to his academic achievements, Professor Allen made significant, regular, and sustained contributions to international professional scientific organisations that represent medical, biomedical and nuclear physics. He was a major international player in the development of medical physics.

Professor Barry Allen was elected President of the

- International Society for Neutron Capture Therapy in 1988,

- Australasian College of Physical Scientists \& Engineers in Medicine (ACPSEM) in 1998,

- Asia-Pacific Federation of Medical Physics (AFOMP) in 2003 ,

- International Organisation for Medical Physics in 2006 (IOMP) and

- International Union of Physical \& Engineering Sciences in Medicine (IUPESM) in 2009.

During his time in various leadership on international medical physics organisations Professor Allen has been instrumental in facilitating a number of initiatives, as well as providing impetus and shouldering the inevitable workload that comes from such roles. On behalf of the ACPSEM, Professor Allen led the successful bid and convened the 2003 World Congress on Medical Physics and Biomedical Engineering, in Sydney.

Professor Allen facilitated the formation of the Vietnamese Association for Medical Physics (VAMP), which hosted the 2008 AFOMP meeting. Cooperating with medical physicists and local hospitals, Professor Allen started an initiative for improved health care in the Mekong Delta. A year later Professor Allen convened the first workshop on palliative radiotherapy for developing countries in Saigon.

Professor Allen was elected Vice-President (PresidentElect) of IOMP in 2003. He was President of IOMP in the period 2006 to 2009. In keeping with furthering the IOMP policy of regional groups, Professor Allen took affirmative action to form the Middle East Federation of Organisations for Medical Physics (MEFOMP) and lobbied for the first regional conference, which was held in Shiraz, Iran in 2011. With regard to the IOMP, he developed a new look Medical Physics World, putting more emphasis and colour on scientific achievements and less on the organisation itself. Professor Allen established the Health Technology Task Group (HTTG) of the IUPESM to assist developing countries in the implementation of appropriate technology and was the inaugural Chair, left lasting positive legacies for the profession.

In 2015 Professor Barry Allen was appointed an Officer in the Order of Australia in the Queen's Birthday honours list. The citation reads "for distinguished service to biomedical physics, particularly to radiation oncology and the development of innovative methods of cancer treatment, and to international professional scientific organisations".

With space, Professor Allen worked in Australia and overseas in top research centers. With time, his research answered current and future needs. With sprit, his passion and leadership, grace and grit has won him esteemed reputation in the medical physics fraternity. He left deep footprints in the space, time and sprit.

Professor Barry J Allen will be missed by many people.

Publisher's Note Springer Nature remains neutral with regard to jurisdictional claims in published maps and institutional affiliations. 